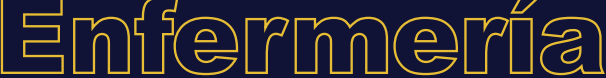 \\ universitaria
}

\section{Calidad de Vida en el trabajo en profesores de colegios públicos de Concepción, Chile}

\section{Quality of Life at work among professors in public colleges in Concepción, Chile}

\section{Qualidade de Vida no trabalho em professores de escolas públicas de Concepción, Chile}

\author{
M. A. Canales-Vergaraa, ${ }^{\mathrm{a},}$, S.V.Valenzuela-Suazo ${ }^{\mathrm{b}}$, L. H. Luengo-Machuca ${ }^{\mathrm{b}}$ \\ ${ }^{a}$ Universidad San Sebastián, Provincia de Concepción, Chile \\ ${ }^{\mathrm{b}}$ Universidad de Concepción, Provincia de Concepción, Chile \\ Recibido: 14 febrero 2018 \\ Aceptado: 26 agosto 2018
}

\section{Resumen}

Objetivo: Medir la Calidad de Vida en el trabajo (CVT) y sus factores asociados en profesores de colegios públicos de Concepción, Chile.

Método: Investigación cuantitativa, transversal, descriptiva. Muestra: 92 profesores. Instrumentos: Se empleó CVT-GOHISALO para evaluar CVT (Alpha de Cronbach 0.952) y un cuestionario biosociodemográfico diseñado por el primer autor. El procesamiento de datos se realizó con SPPS, empleándose Rho-Spearman, Rho-Pearson, ANOVA y pruebas no-paramétricas. Se contó con las autorizaciones de los comités de ética involucrados, así como de la Dirección de Administración de Educación Municipal de Concepción (DAEM), y con el consentimiento informado de los participantes.

Resultados: Promedio CVT global: 215.8 (DE=43.3), que indica nivel medio de CVT.

Promedios CVT por dimensiones: Soporte institucional para el trabajo (SIT) $=44.6(\mathrm{DE}=10.2)$, Seguridad en el trabajo (ST)=30 (DE=11.7), Integración al puesto de trabajo (IPT)=33.4 (DE=6.1), Satisfacción por el trabajo $(\mathrm{SPT})=34.9(\mathrm{DE}=6.8)$, Bienestar logrado a través del trabajo $(\mathrm{BAT})=36.6(\mathrm{DE}=5.7)$, Desarrollo personal $(\mathrm{DP})=23.3(\mathrm{DE}=6.4)$ y Administración del tiempo libre $(\mathrm{ATL})=12.7(\mathrm{DE}=4.2)$. Clasificación CVT por dimensiones: SIT: nivel alto; ST, IPT, SPT y DP: nivel medio; BAT y ATL: nivel bajo. Anova para prexistencia de trastorno en salud mental y CVT global, dimensiones SIT, ST, IPT, SPT, BAT, DP y ATL con valor $p=0.000 ; p=0.000 ; p=0.017 ; p=0.005 ; p=0.000 ; p=0.001$ y $p=0.046$ respectivamente.

Conclusiones: Los maestros se encuentran medianamente satisfechos con su CVT; existe asociación entre prexistencia de trastornos en salud mental y baja percepción de CVT. Es fundamental que enfermería vele por adecuadas condiciones laborales de profesores y alumnos con el fin de asegurar una CVT satisfactoria así como mejorar la calidad de la educación impartida.

\footnotetext{
* Autor de correspondencia. Correo electrónico: marcelo.canalesv@docente.uss.cl http://dx.doi.org/10.22201/eneo.23958421e.2018.4.544 
Palabras clave: Calidad de Vida; maestros; /educación; trabajo; Chile.

\section{Abstract}

Objective: To assess the Quality of Life at Work (QLW) and associated factors among professors of public colleges in Concepción, Chile.

Method: This is a quantitative, transversal, and descriptive study using a sample of 92 professors. The CVT-GOHISALO instrument was administered to assess QLW (Cronbach Alpha $=0.952$ ) in addition to a bio-social-demographic questionnaire specifically designed by the first author. Data were analyzed with SPSS calculating Rho-Spearman, Rho-Pearson, ANOVA, and non-parametric tests. The corresponding authorizations from involved ethics committees and the Direction of Management of Municipal Education of Concepcion were obtained as well as the inform consent from all the participants.

Results: The global average QLW score was 215.8 ( $\mathrm{SD}=43.3$ ), indicating a medium level. The related average scores by dimension were as follows: Institutional Support for Work (ISW) $=30 \quad(\mathrm{SD}=11.7)$; Work Security $(\mathrm{WS})=30(\mathrm{SD}=11.7)$; Integration to the Work Position (IWP)=33.4 (SD=6.1); Work Satisfaction (WS)=34.9 (DS=6.8); Wellbeing Achieved from Work (WAW)=36.6 (DS=5.7); Personal Development $(\mathrm{PD})=23.3(\mathrm{SD}=6.4)$; and Free Time Management $(\mathrm{FTM})=12.7(\mathrm{SD}=4.2)$. The QLW classification by dimension is as follows: ISW=high; WS, IWP, WS, and PD=medium; WAW, and FTM=low. ANOVA for pre-existing mental health issues and global QLV, dimensions ISW, WS, IWP, WS, WAW, PD, FTM had $p=0.000 ; p=0.000 ; p=0.017 ; p=0.005 ; p=0.000 ; p=0.001$ and $p=0.046$ respectively.

Conclusions: Teachers are satisfied in a medium level regarding their QLW. An association between preexisting mental health issues and a low perception of QLW was found. It is fundamental that nursing organizations promote adequate working conditions for professors and students in order to foster their satisfaction at work and thus the quality of the education they impart.

Keywords: Quality of Life; school teachers; /education; work; Chile.

\section{Resumo}

Objetivo: Medir a Qualidade deVida no trabalho (CVT) e seus fatores associados em professores de escolas públicas de Concepción, Chile.

Método: Pesquisa quantitativa, transversal, descritiva. Amostra: 92 professores. Instrumentos: Empregou-se CVT-GOHISALO para avaliar CVT (Alpha de Cronbach 0.952) e um questionário biosociodemográfico desenhado pelo primeiro autor. O processamento de dados realizou-se com SPPS, empregou-se Rho-Spearman, Rho-Pearson, ANOVA e provas não-paramétricas. Contou-se com as autorizações dos comités de ética envolvidos, assim como da "Dirección de Administración de Educación Municipal de Concepción" (DAEM), e com o consentimento informado dos participantes.

Resultados: Média CVT global: 215.8 ( $\mathrm{DE}=43.3$ ), que indica nível médio de CVT. Média CVT por dimensões: Suporte institucional para o trabalho $(\mathrm{SIT})=44.6(\mathrm{DE}=10.2)$, Segurança no trabalho $(\mathrm{ST})=30$ (DE=11.7), Integração ao posto de trabalho (IPT) $=33.4(\mathrm{DE}=6.1)$, Satisfação pelo trabalho $(\mathrm{SPT})=34.9$ $(\mathrm{DE}=6.8)$, Bem-estar atingido a través do trabalho (BAT)=36.6 ( $\mathrm{DE}=5.7)$, Desenvolvimento pessoal $(D P)=23.3(D E=6.4)$ e Administração do tempo livre (ATL)=12.7 (DE=4.2). Classificação CVT por dimensões: SIT: nível alto; ST, IPT, SPT e DP: nível médio; BLT e ATL: nível baixo. Anova para preexistência de transtorno em saúde mental e CVT global, dimensões ST, IPT, SPT, BAT, DP e ATL com valor p=0.000; $\mathrm{p}=0.000 ; \mathrm{p}=0.017 ; \mathrm{p}=0.005 ; \mathrm{p}=0.000 ; \mathrm{p}=0.001$ e $\mathrm{p}=0.046$, respectivamente.

Conclusões: Os professores encontram-se medianamente satisfeitos com sua CVT; existe associação entre preexistência de transtornos em saúde mental e baixa percepção de CVT. É fundamental que a enfermagem garanta adequadas condições laborais de professores e alunos com o fim de segurar uma CVT satisfatória assim como melhorar a qualidade da educação ministrada.

Palavras chave: Qualidade de Vida; professores; /educação; trabalho; Chile. 


\section{Introducción}

La Calidad de Vida en el trabajo (CVT) constituye un término multidimensional cuyos cimientos reposan en un conjunto de creencias, que abarcan desde los esfuerzos por incrementar la productividad, promover la moral, participación, dignidad y motivación de los trabajadores, hasta el hecho de eliminar los aspectos disfuncionales de la jerarquía organizacional ${ }^{1}$. Esto cobra relevancia si se considera que el trabajo es la actividad a la que se dedica mayor cantidad de tiempo, en este caso, la calidad de vida es un factor directamente relacionado con el grado de salud/enfermedad ${ }^{2}$. Asimismo, la CVT constituye una variable que tiene directa repercusión en el rendimiento laboral, ausentismo y otra serie de fenómenos que facilitan el deterioro de la eficiencia organizacional, lo que origina pérdida de recursos económicos y aumento en gastos asociados ${ }^{3}$, circunstancias que en el caso de los maestros, repercuten en la calidad de la educación/enseñanza impartida, de modo, que la medición de la CVT contribuiría a mejorar los ambientes laborales a través de la promoción del trabajo decoroso y el aumento de la eficiencia del proceso educativo.

Sobre la CVT de los profesionales dedicados a la docencia en educación básica y enseñanza media (preparatoria), poco se conoce, aunque, varias investigaciones realizadas a nivel latinoamericano entre los años 1990 a 2017, tienen como elemento común que los maestros están sometidos a condiciones de trabajo precarias, marcadas por la sobrecarga laboral, excesivo número de alumnos por curso, jornadas laborales extensas muchas veces no reconocidas ni remuneradas, bajos salarios, discriminación social y bajo o escaso reconocimiento, ambientes laborales vulnerables marcados por la violencia y el narcotráfico. A su vez, los establecimientos educativos, en gran medida no cuentan con la infraestructura, personal, recursos didácticos y económicos necesarios para llevar a cabo el proceso educativo ${ }^{4-11}$. Este contexto da cuenta que existen graves falencias que afectan directa o indirectamente la CVT, hecho relevante si se considera que el proceso enseñanza - aprendizaje sólo puede ser efectivo si aquellos que lo imparten, es decir, los profesores, lo hacen bajo condiciones adecuadas ${ }^{12}$.

En tanto, Chile destaca por presentar una realidad docente con aspectos críticos, tales como la carencia de espacios sanitarios adecuados, mobiliario inadecuado, material de trabajo insuficiente y no apropiado, lo que genera sobrecarga ergonómica y aporte informal de recursos por los propios educadores. Por otro lado, las condiciones sociales de trabajo destacan por la presencia de violencia, amenazas y situaciones de riesgo ${ }^{5}$. La falta de apoyo por parte de los actores del proceso educativo constituye un factor de sobrecarga para la realización de la tarea pedagógica, a lo que se le suma el excesivo número de alumnos por curso y una jornada laboral que muchas veces demanda gran cantidad de tiempo en tareas pedagógicas fuera del horario de trabajo, esto genera la invasión del trabajo docente hacia el espacio de descanso y doméstico ${ }^{5}$. En este sentido, Chile posee un perfil docente que da cuenta de un malestar relacionado, principalmente, con la prevalencia de enfermedades crónicas, alta exigencia ergonómica y desgaste de salud mental ${ }^{5}$, de modo que dar respuesta a estas circunstancias se convierte en una imperante necesidad que, también debe abarcar el desarrollo de reformas educacionales, que además de dar solución a los problemas relacionados con la calidad de la misma, velen y promuevan la salud docente, pues es imposible concebir mejoras en la calidad educativa sin mejorar la CVT.

En este sentido, la presente investigación, bajo el contexto de salud ocupacional, pretende visibilizar el grado de satisfacción percibido respecto a la situación laboral vivenciada por el estamento docente, para apoyar en el fomento de la creación de ambientes laborales saludables y contribuir en la elaboración de políticas educativas, así como de salud pública con el propósito de conservar y fomentar la salud del profesorado.

Para abordar la problemática, se utilizará una perspectiva teórico - práctica que analiza la CVT como un constructo conformado por una dimensión subjetiva y otra objetiva ${ }^{1}$, la primera reúne aspectos tales como: satisfacción de necesidades, satisfacción laboral, relaciones interpersonales con la media y alta gerencia, actitudes, además de valores hacia el trabajo como: motivación, compromiso, orgullo, identificación; la segunda se compone de aspectos asociados a las condiciones del entorno laboral como: 
seguridad, iluminación, ergonomía, tecnología, etc., además de aquellas relacionadas con el puesto de trabajo, con los sistemas de gestión, así como las características y procesos organizacionales ${ }^{1,3}$. De esta manera, se espera describir los factores que inciden en la percepción de CVT mediante el Modelo de Sistemas de Betty Neuman, el cual posibilita la ejecución de un análisis multivariado, a través de la inclusión de variables de tipo cultural, social, biológicas, económicas y psicológicas, que conforman el entorno de la profesión docente ${ }^{13}$.

Bajo este contexto es que Enfermería como ciencia social y en congruencia a la legislación chilena, es la disciplina encargada de velar por la gestión del cuidado, y como tal, de ejercer acciones concretas para la prevención y promoción de la salud. Dicha labor, en palabras del Consejo Internacional de Enfermeras (CIE), también involucra cultivar la salud ocupacional de los trabajadores, y promover ambientes laborales saludables ${ }^{14}$, la presente investigación es soporte para ello, al contribuir a la mejora en la calidad de vida en el trabajo del profesorado, con lo que se fomentaría la salud de dicho estamento, hecho que favorecerá la calidad de la enseñanza impartida hacia la comunidad ${ }^{4}$.

\section{Metodología}

Investigación cuantitativa, descriptiva, transversal y correlacional ${ }^{15}$. La unidad de análisis fueron profesores de colegios o liceos públicos de la ciudad de Concepción, Chile. La población la conforman 1051 profesores, distribuidos en 32 colegios y liceos públicos de la ciudad de Concepción, Chile ${ }^{16,17}$. La muestra fue seleccionada a través de muestreo probabilístico estratificado, cada establecimiento educacional constituyó un estrato, se consideraron 3 educadores por colegio/liceo, para un total de 96 profesores, de los cuales, cuatro no participaron, por lo que la muestra final fue de 92. La probabilidad de participar se expresó en la medida en que se incluyó en la muestra, sólo a aquellos profesores que al azar estuvieron disponibles al momento que se visitó las dependencias educativas.

Criterio de inclusión: Profesores con al menos seis meses de estar laborando en el colegio o liceo; los de exclusión: estar con permiso administrativo o licencia médica durante la aplicación de la encuesta.

La muestra fue calculada mediante el software Infostat para un intervalo de confianza de 5, fue recabada entre los meses de agosto y diciembre de 2016, en los colegios y liceos de la ciudad de Concepción, provincia del Bio-Bio, Chile, mediante encuesta autoaplicada, la que podía responderse en el trabajo u hogar, para posteriormente entregarla sellada en un sobre al investigador en forma directa. Cabe destacar que mediante preguntas incluidas en el cuestionario biosociodemográfico, se cuidó que ningún docente que trabajara en dos o más establecimientos incluidos en esta investigación, duplicara su encuesta.

Se realizó prueba piloto en tres establecimientos educacionales administrados por la Dirección de Administración Municipal (DAEM) de Concepción, Chile, se contó con la participación de nueve educadores, quienes refirieron sugerencias al formato técnico de las encuestas, las que fueron consideradas para su rediseño.

El objetivo general fue abordado a través de la aplicación de la encuesta sociodemográfica y del instrumento para medir calidad de vida en el trabajo CVT-GOHISALO ${ }^{18,19}$, creado y validado en México por González et a ${ }^{18}$; aplicado a médicos y enfermeras(os) de tres niveles de atención durante el año 2010. Posee una validez total de 0.6777 y una confiabilidad de $0.9527^{19}$, y fue validado en Chile mediante la determinación de cinco jueces. Cabe destacar que en la presente investigación dicho instrumento mostró una consistencia interna de 0.971 .

CVT-GOHISALO se compone de 74 ítems divididos en siete dimensiones: 1) soporte institucional para el trabajo (SIT), 2) seguridad en el trabajo (ST), 3) integración al puesto de trabajo (IPT), 4) satisfacción por el trabajo (SPT), 5) bienestar a través del trabajo (BAT), 6) desarrollo personal (DP) y 7)administración tiempo libre (ATL), cada una de las cuales puede ser calificada de forma individual o en conjunto $^{19,20}$ (Tabla 1). Cada item se evalúa con escala tipo Likert con puntuación de 0 a 4 , el cero corresponde al mínimo de satisfacción/ acuerdo/ frecuencia/ compromiso y el cuatro al máximo de cada valoración. 
Tabla 1. Puntajes de Corte CVT-GOHISALO

\begin{tabular}{lccc}
\hline Dimensión & Baja & Media & Alta \\
\hline Soporte institucional (SIT) & $17-28$ & $29-37$ & $38-56$ \\
Seguridad en el trabajo (ST) & $6-23$ & $24-36$ & $37-58$ \\
Integración al puesto de trabajo (IPT) & $24-29$ & $30-34$ & $35-40$ \\
Satisfacción por el trabajo (SPT) & $28-33$ & $34-38$ & $39-44$ \\
Bienestar logrado a través del trabajo (BAT) & $34-36$ & $37-39$ & $40-44$ \\
Desarrollo personal del trabajador (DP) & $16-21$ & $22-26$ & $27-32$ \\
Administración del tiempo libre (ATL) & $12-14$ & $15-17$ & $18-20$ \\
Calidad de Vida en el Trabajo global(CVT) & $56-191$ & $192-227$ & $228-296$ \\
\hline
\end{tabular}

Fuente: González et al ${ }^{19}$

Los autores de dicho instrumento refieren que dadas sus características sería posible emplearlo en todo tipo de trabajadores o rubros ${ }^{19}$.

Para el procesamiento de datos fue empleado el software estadístico Microsoft Excel y SPSS v15. Se utilizaron estadísticos descriptivos, tales como; moda, media, frecuencias, mediana, desviación estándar; para el análisis de asociaciones entre CVT, sus dimensiones y las variables biosociodemográficas, se utilizó ANOVA, Rho de Spearman, Rho de Pearson y la U de Mann-Whitney.

El estudio fue aprobado por el Comité de Ética de la Facultad de Enfermería de la Universidad de Concepción, Chile, organismo que refiere no tener conflicto de intereses. Igualmente, se contó con el consentimiento informado de cada uno de los participantes y autorización por parte de la Dirección de Administración de Educación Municipal (DAEM).

Es importante recalcar que la investigación se desarrolló teniendo en cuenta los siete requisitos éticos propuestos por Ezekiel Emanuel ${ }^{21}$, de este modo, la investigación aporta en la creación y fomento de espacios laborales saludables para el magisterio; posee objetivos medibles y alcanzables; a todos los profesores se les dio la misma posibilidad de participar en la investigación; todos los participantes fueron informados de los beneficios del estudio; mientras que a los riesgos se les dio respuesta mediante asistencia psicológica por parte de CAPSIS U de C.

Es preciso mencionar que se contó con la autorización de los autores del instrumento CVT-GOHISALO; una vez concluida y aprobada la investigación se entregaron los resultados tanto a la DAEM de Concepción, como a cada uno de los colegios y liceos públicos donde se desarrolló el estudio, se resguardó de forma cuidadosa la identidad, confidencialidad y privacidad de cada uno de los participantes.

Los investigadores confirman no tener conflicto de intereses.

\section{Resultados}

En cuanto a las variables biosociodemográficas destaca que el 32.6\% de los docentes encuestados laboraban en establecimientos educacionales que otorgaban enseñanza parvularia, básica y media, el $22.8 \%$ de primero a octavo básico y el $15.2 \%$ de primero a cuarto medio. La media de edad de la muestra fue de 40.8 años, el mínimo fue 22 años y el máximo 64 años; el 81.5\% de la muestra eran mujeres; el 66.3\% de los sujetos se encontraba en una relación de pareja; la media de años en ejercicio de la docencia fue de 13.8 años. Por otra parte, la religión más profesada fue la católica con un 58.7\%, seguida de la evangélica con $21.7 \%$.

Respecto a indicadores epidemiológicos de la muestra, destaca que el $29.3 \%$ de los profesores padecía alguna enfermedad crónica, la hipertensión arterial es la de mayor prevalencia, esta afecta a un $9.8 \%$ del total; le sigue el asma con un $7.6 \%$ y la diabetes mellitus tipo 2 con un $3.3 \%$. En cuanto a salud mental, sólo el 15.2\% refirió haber sido diagnosticado con algún trastorno en dicha área, de los cuales, la depresión y el estrés laboral fueron los únicos descritos, la primera se destaca por ser la más reportada con una prevalencia de vida de un $14.1 \%$ para el total de sujetos. 
En cuanto al perfil laboral docente, solo el 19.6\% de la muestra tenía más de un empleo; el 64\% laboraba de forma presencial más de 30 horas semanales y un 25\% lo hacía más de 40 horas; la media de horas de trabajo presencial fue de 33.9 a la semana, el mínimo fue de dos horas y el máximo de 50. El $44.6 \%$ de los profesores refirió haber hecho uso de licencia médica durante el último año; del total que hicieron uso, el $51.2 \%$ fue por un periodo de 1 a 5 días, el $24.4 \%$ de 6 a 15 días, $12.2 \%$ por un periodo de 16 a 30 días y otro $12 \%$ por más de 30 días. Cabe destacar que el $44.6 \%$ de los profesores refirió haber sido víctima de violencia laboral alguna vez en su vida.

Sobre el descanso durante la jornada laboral, solo el 6.5\% de los maestros no destinaba tiempo para realizar pausas, el $29.3 \%$ utilizaba diariamente entre 5 a 15 minutos, el $26.1 \%$ de 16 a 30 minutos, el $22.8 \%$ de 31 a 45 minutos, el $10.9 \%$ entre 46 a 60 minutos y solo el $4.3 \%$ utilizaba más de 60 minutos.

La Tabla 2 presenta los resultados sobre el nivel de CVT percibido por la muestra, la CVT global es valorada como media. De igual modo, cuatro de las siete dimensiones: Seguridad en el trabajo (ST), Integración al puesto de trabajo (IPT), Satisfacción por el trabajo (SPT) y Desarrollo personal (DP) percibidas con una CVT media; mientras que dos dimensiones: Bienestar logrado a través del trabajo (BAT) y Administración del tiempo libre (ATL) con una CVT baja; y solo una: Soporte institucional para el trabajo (SIT) con una CVT alta.

En cuanto a la relación entre variables, se detectó mediante ANOVA (Tabla 3) y U de Mann-Whitney (Tabla 4), asociación estadísticamente significativa entre Calidad de vida en el trabajo Global y Prexistencia de trastorno en salud mental, esto establece que obtener un bajo puntaje en la CVT global se asociaría a haber sido diagnosticado con alguna patología en salud mental (depresión) en algún momento de la vida. Cabe destacar que para dicho entrecruzamiento se desestimó la respuesta estrés laboral, debido a que solo se documentó un caso.

Tabla 2. Puntaje, calificación y percepción de CVT global y por dimensión ( $n=92)$

\begin{tabular}{lccccc}
\hline Dimensión CVT & $\begin{array}{c}\text { Puntaje } \\
\text { promedio }\end{array}$ & $\begin{array}{c}\text { Calificación } \\
\text { promedio }\end{array}$ & $\begin{array}{c}\text { Percepción } \\
\text { alta (\%) }\end{array}$ & $\begin{array}{c}\text { Percepción } \\
\text { media (\%) }\end{array}$ & $\begin{array}{c}\text { Percepción } \\
\text { baja (\%) }\end{array}$ \\
\hline Global & 215.8 & CVT media & $42(45.7)$ & $24(26.1)$ & $26(28.3)$ \\
Soporte institucional para el trabajo (SIT) & 44.6 & CVT alta & $73(79.3)$ & $11(12.0)$ & $8(8.7)$ \\
Seguridad en el trabajo (ST) & 30.0 & CVT media & $30(32.6)$ & $35(38.0)$ & $27(29.3)$ \\
Integración al puesto de trabajo (IPT) & 33.4 & CVT media & $51(55.4)$ & $20(21.7)$ & $21(22.8)$ \\
Satisfacción por el trabajo (SPT) & 34.9 & CVT media & $30(32.6)$ & $25(27.2)$ & $37(40.2)$ \\
Bienestar logrado a través del trabajo (BAT) & 36.6 & CVT baja & $31(33.7)$ & $21(22.8)$ & $40(43.5)$ \\
Desarrollo personal (DP) & 23.3 & CVT media & $32(34.8)$ & $28(30.4)$ & $32(34.8)$ \\
Administración del tiempo libre (ATL) & 12.7 & CVT baja & $10(10.9)$ & $24(26.1)$ & $58(63.0)$ \\
\hline
\end{tabular}

Tabla 3. Anova para Calidad de vida en el trabajo Global y Prexistencia de trastorno de salud mental

\begin{tabular}{|c|c|c|c|c|c|}
\hline & & gl & Media cuadrática & $\mathbf{F}$ & Sig. \\
\hline $\begin{array}{l}\text { Puntaje global calidad de vida en el trabajo * } \\
\text { Prexistencia de trastorno de salud mental }\end{array}$ & Entre grupos & 1 & 25975.744 & 16.164 & .000 \\
\hline
\end{tabular}

Tabla 4. U de Mann-Whitney para Calidad de vida en el trabajo Global y depresión

\begin{tabular}{lr}
\hline \multicolumn{2}{c}{ Puntaje global calidad de vida en el trabajo } \\
\hline U de Mann-Whitney & 201.000 \\
$Z$ & -3.471 \\
Sig. asintótica (bilateral) & $\mathbf{. 0 0 1}$ \\
\hline${ }^{*} \leq 0.05$ &
\end{tabular}


También, existió asociación estadísticamente significativa entre las dimensiones Seguridad en el trabajo, Integración al puesto de trabajo, Satisfacción por el trabajo, Bienestar logrado a través del trabajo, Desarrollo personal, Administración del Tiempo libre con la variable Prexistencia de trastorno de salud mental (Tabla 5), es decir, cuando existe menor satisfacción en dichas dimensiones, los sujetos presentan antecedentes de haber sido diagnosticados con depresión alguna vez en la vida.

Tabla 5: Anova para dimensiones de calidad de vida en el trabajo y Prexistencia de trastorno de salud mental

\begin{tabular}{|c|c|c|c|c|c|}
\hline & & gl & $\begin{array}{l}\text { Media } \\
\text { cuadrática }\end{array}$ & $\mathbf{F}$ & Sig. \\
\hline Seguridad en el trabajo * Prexistencia de trastorno de salud mental & Entre grupos & 2 & 1133.542 & 9.827 & .000 \\
\hline Integración al puesto de trabajo * Prexistencia de trastorno de salud mental & Entre grupos & 2 & 149.947 & 4.279 & .017 \\
\hline Satisfacción por el trabajo * Prexistencia de trastorno de salud mental & Entre grupos & 2 & 241.375 & 5.646 & .005 \\
\hline $\begin{array}{l}\text { Bienestar logrado a través del trabajo * Prexistencia } \\
\text { de trastorno de salud mental }\end{array}$ & Entre grupos & 2 & 266.092 & 9.637 & .000 \\
\hline Desarrollo personal * Prexistencia de trastorno de salud mental & Entre grupos & 2 & 293.226 & 8.191 & .001 \\
\hline $\begin{array}{l}\text { Administración del Tiempo libre * Prexistencia } \\
\text { de trastorno de salud mental }\end{array}$ & Entre grupos & 2 & 53.890 & 3.179 & .046 \\
\hline
\end{tabular}

Finalmente, se detectó asociación entre la dimensión Bienestar logrado a través del trabajo y la variable Uso de licencia médica durante el último año (Tabla 6), lo que se traduce en que aquellos docentes que hicieron uso de licencia médica durante el último año, presentaron menores niveles de CVT en dicha dimensión.

Tabla 6: Anova para la dimensión Bienestar logrado a través del trabajo y Uso de licencia médica durante el último año

\begin{tabular}{|c|c|c|c|c|c|}
\hline & & gl & Media cuadrática & $\mathbf{F}$ & Sig. \\
\hline $\begin{array}{l}\text { Bienestar logrado a través del trabajo * Uso de } \\
\text { licencia médica durante el último año }\end{array}$ & Entre grupos & 1 & 130.790 & 4.118 & .045 \\
\hline
\end{tabular}

\section{Discusión}

Respecto al tipo de establecimiento donde se ejerce la docencia, existe poca evidencia que dé cuenta sobre la diferencia en cuanto a las condiciones de trabajo entre los distintos niveles de enseñanza, sin embargo, algunos estudios establecen que para los educadores de enseñanza básica tendrían bajos niveles de estrés ${ }^{22}$, mientras que para los de enseñanza media existiría mayor frecuencia de sintomatología asociada a burnout ${ }^{23}$. Esta situación sugiere que el nivel de enseñanza donde se desempeñan los pedagogos podría afectar la CVT percibida, no obstante, la presente investigación no pudo validar dicha idea.

Por otro lado, tanto el predominio del sexo femenino como el hecho de tener pareja, coinciden con los datos del primer Censo Docente en Chile $2012^{8}$ y con la investigación de la OREALC/UNESCO en Latinoamérica sobre sobre salud docente ${ }^{5}$, esto es que debido a su quehacer implicaría un triple rol, es decir, ser madre, pareja y trabajadora a la vez, circunstancia que lógicamente podría contribuir a un mayor deterioro de su salud ${ }^{24}$. A esto se agrega las diferencias de ingresos entre hombres y mujeres, hecho que puede afectar la salud mental docente se puede generar frustración y detrimento en la calidad de la educación impartida ${ }^{25}$.

La variable edad también mostró un comportamiento similar a lo censado por el Ministerio de Educación de Chile (MINEDUC) ${ }^{26}$ y por la OREALC/UNESCO para educadores Chilenos ${ }^{5}$; a pesar de ello, la media de años en ejercicio de la docencia fue un poco menor a lo señalado por el MINEDUC ${ }^{26}$, quienes sitúan dicho promedio en 16 años, y a la vez un poco mayor a lo planteado por la OREALC/UNESCO 
para Chile, quienes la sitúan en 10 años ${ }^{5}$. Ambos indicadores dan cuenta de una población relativamente madura, probablemente con una mayor prevalencia de patologías crónicas y/o factores de riesgo y mayor experiencia en la labor educativa ${ }^{27}$.

En cuanto a religión, los datos de la presente investigación fueron similares a los del primer Censo Docente en Chile $2012^{8}$, donde católicos y evangélicos fueron mayoría con un $87 \%$ y $10 \%$, respectivamente, situación que puede aportar comprensión en torno al apoyo espiritual percibido por los educadores $^{28}$.

Respecto a las Enfermedades Crónicas No Transmisibles (ECNT), la hipertensión arterial resultó ser la principal detectada, aunque la prevalencia fue menor que la media nacional (26.9\%) y que la detectada por la OREALC/UNESCO con un $38 \% \%^{5}$, hecho que sugiere la presencia de una población docente relativamente sana, con baja incidencia de patologías crónicas, hecho que se contrapone con la mayoría de los sondeos sobre salud docente, aunque éstos dan cuenta principalmente de patologías de origen ocupacional como disfonías o afonías o referentes a lo psicosomático ${ }^{5}$.

En relación a salud mental, el principal trastorno descrito con mayor prevalencia fue la depresión con un $14.1 \%$, cifra muy por debajo a lo reportado por la OREALC/UNESCO y la Encuesta Nacional de Salud 2009-2010, las que dan cuenta de cifras mayores, que ascienden a $25.9 \%{ }^{5}$ y $21 \%^{29}$, respectivamente. Cabe destacar que el presente estudio tampoco reportó asociación entre ser docente y una mayor probabilidad de padecer depresión, aunque son muchas las investigaciones que describen como real dicha asociación $^{5-7}$. Es este trabajo, dicha inconsistencia podría tener origen en diversos factores que abarcarían desde deseabilidad social hasta la ausencia o exclusión de la muestra de aquellos maestros que estaban con licencia médica por causales asociadas a salud mental.

Si bien el multiempleo era muy cercano al $20 \%$ reportado por otras investigaciones chilenas 5 , fue considerablemente más bajo que lo planteado por Cornejo-Chávez en 2009, cuya cifra era cercana al $50 \%$. Esta situación podría relacionarse con las bajas remuneraciones percibidas por los maestros ${ }^{5-8}$, las que según datos del MINEDUC ascienden a 591.536 pesos (bruto) al quinto año de egreso ${ }^{26}$, muy por debajo de lo esperado. Por si fuera poco, según datos del primer Censo Docente en Chile $2012^{8}$, más de un tercio de los encuestados (36\%) se encontraba bajo condiciones laborales inestables, hecho que además de generar incertidumbre laboral y multiempleo, afectaría la salud mental de los educadores.

En esta investigación, el número de horas trabajadas semanalmente es menor a lo planteado por la OREALC/UNESCO ${ }^{5}$, quienes estiman que, en Chile casi el $60 \%$ de los profesores trabajan más de 40 horas a la semana, mientras que el $80 \%$ trabaja más de 30 horas a la semana, asimismo, el primer Censo Docente en Chile $2012^{8}, 46 \%$ de los educadores trabaja entre 41 y 46 horas semanales, mientras que el $77 \%$ lo hace más de 30 horas. El elevado número de horas trabajadas a la semana puede relacionarse con sobrecarga laboral, falta de docentes contratados o la necesidad de obtener mayores remuneraciones, ya que frecuentemente éstas son descritas como insuficientes ${ }^{8}$. También, es preciso agregar el trabajo no remunerado realizado en casa, el que ha sido reportado por diversas investigaciones ${ }^{5-8}$, lo que significa que casi un tercio de los docentes labore en su hogar un promedio de 10 a 12 horas adicionales a su jornada ${ }^{8}$. De este modo, dichas circunstancias no sólo limitarían la vida de los educadores, sino que además generarían estrés y agotamiento físico, el cual se somatizaría y cronificaría.

En este trabajo, se encontró que poco más de dos quintas partes de los maestros afirma haber sido víctima de violencia laboral alguna vez en su vida, converge con lo manifestado por la OREALC/UNESCO ${ }^{5}$ y Cornejo-Chávez ${ }^{7}$, quienes sostienen que al menos un tercio de los educadores ha sufrido violencia en su lugar de trabajo. En este sentido, la elevada cifra de violencia experimentada por los maestros es fuente de agotamiento emocional, lo que sumado a las extensas jornadas de trabajo constituyen una verdadera bomba de tiempo para trastornos en salud mental, hecho que no solo dañaría al profesor, sino que también tendría un consecuente deterioro de la calidad de la educación impartida ${ }^{30}$.

En cuanto al tiempo destinado a descanso durante la jornada laboral, este estudio difiere con lo referido por la OREALC/UNESCO $/{ }^{5}$ en docentes chilenos, donde el $31.7 \%$ de los maestros hacia pausas 
por menos de 5 minutos diarios, el $12 \%$ de 5 a 15 minutos, el $27.7 \%$ de 15 a 30 minutos y el $28.6 \%$ más de 30 minutos. El principal contraste se produce en los extremos de tiempo, por una parte, en la presente investigación, eran muy pocos los maestros que no se tomaban una pausa durante su jornada laboral, mientras que la mayoría ocupaban más de 30 minutos diarios para ello, del mismo modo, la OREALC/UNESCO ${ }^{5}$ muestra una distribución más uniforme en los tiempos destinados a pausa. Algunos autores como Cornejo-Chávez ${ }^{7}$, sugieren que las condiciones materiales presentes en los establecimientos educacionales junto a la sobrecarga laboral jugarían en contra al momento de realizar pausas durante la jornada de trabajo ${ }^{7}$, de igual manera, la OREALC/UNESCO $/{ }^{5}$ sostiene que menos de 15 minutos diarios de descanso durante la jornada laboral sería un factor de riesgo para la salud docente, cifra que en el presente estudio abarcaría a un $35.8 \%$ de los profesores, dichos resultados podrían asociarse a que, en el caso de estos últimos, existiera sobrecarga que les impidiera tomarse un tiempo para descansar, o bien las jefaturas les negarían el descanso. Por otra parte, puede ser que aquellos que tomaran más de 30 minutos diarios, se encontraran profundamente agobiados, o que las jefaturas no realizaran una supervisión adecuada.

Respecto al uso de licencia médica durante el último año, éste fue similar a lo reportado por la OREALC/UNESCO 5 y por el Estudio de Salud Laboral de los Profesores, ambos para Chile, con cifras de $46.5 \%$ y $45.5 \%{ }^{6}$, respectivamente.

Si bien existió un alto uso de licencia médicas, la mayoría de éstas fueron de corta duración, hecho que la literatura relaciona con la mayor exposición de los maestros con agentes infecciosos (contacto con grandes grupos de estudiantes) $)^{5}$. Por otro lado, existe consenso de que muchas de las licencias tienen origen en patologías de salud mental vinculadas o no a los altos niveles de estrés experimentados por los docentes, o bien a las manifestaciones somáticas de las mismas ${ }^{5-8}$, de modo que las licencias extendidas por un periodo mayor a 30 días podrían asociarse a estas últimas.

Para la discusión de las asociaciones detectadas entre las variables, solo se hará referencia a aquellas que hayan sido estadísticamente significativas, en este sentido, la asociación encontrada entre la CVT Global y las dimensiones: Seguridad en el trabajo, Integración al puesto de trabajo, Satisfacción por el trabajo, Bienestar logrado a través del trabajo, Desarrollo personal, Administración del Tiempo libre y la variable Prexistencia de Patología en salud mental, hace referencia a que los docentes que habían sido diagnosticados alguna vez en la vida con depresión, presentaban tendencia a percibir con menor satisfacción tanto su CVT Global como cada una de las dimensiones mencionadas, dicho fenómeno podría encontrar explicación en la patogenia propia de la depresión ${ }^{31}$, como a las características innatas del trabajo docente $^{5-7}$, el que de por sí conlleva un desgaste en salud mental, asociándose inclusive a la aparición de cuadros depresivos ${ }^{7,32}$, situación que el modelo de sistemas de Neuman ${ }^{13}$ explica en relación a que los factores que gestionan la asociación entre la CVT Global, sus dimensiones y la prexistencia de trastorno en salud mental, serían estresores que hallarían su raíz en la naturaleza de la profesión docente, los que, debido al exiguo grosor de la línea de resistencia, es decir, la escasa cantidad de recursos disponibles para que el educador pueda defenderse de los factores que producen deterioro de la CVT (estresores), terminan por traspasar la línea normal de defensa, causante de pérdida del bienestar, que sumado a la gran cantidad de energía que debe emplear el maestro para enfrentar los estresores, termina por colapsar su capacidad adaptativa, lo que puede llegar a ocasionar algún tipo de trastorno en salud mental, el que a su vez, de uno u otro modo estará latente mientras las condiciones de trabajo sigan siendo las mismas, hecho que indudablemente, a modo de circulo-vicioso, se verá reflejado en insatisfacción respecto a la CVT Global y sus dimensiones ${ }^{33,34}$.

La asociación encontrada entre la dimensión Bienestar lograda a través del trabajo y la variable uso de licencia médica durante el último año, da cuenta que aquellos docentes que presentaron menores niveles de satisfacción con dicha dimensión, mostraban mayor frecuencia en el uso de licencia médica durante el último año, hecho que resulta esperable si se considera que la mencionada dimensión, hace referencia a aspectos tanto concernientes a la satisfacción con la labor profesional desempeñada como a la salud física 
y mental ${ }^{18}$, que basándose en el modelo de Neuman, daría cuenta que en esta asociación los estresores al traspasar la línea normal de defensa y resistencia, terminan desencadenando uso de licencias médicas, cuyo fin sería el restablecimiento de los límites del sistema-usuario o reconstitución, proceso que se ve modificado por la presencia de factores de índole intrapersonal como la resiliencia, y extrapersonales, como las redes de apoyo y las características propias del ambiente laboral. A su vez, ambos elementos permiten explicar fenómenos paradójicos, la resiliencia es la probable causa de que un gran número de maestros defina la satisfacción con la dimensión Bienestar logrado a través del trabajo, como alta, hecho que se contrapondría a la información encontrada en la literatura ${ }^{29,31}$, por lo tanto, las características propias del ambiente laboral docente, indudablemente contribuyen a la insatisfacción con los componentes de dicha dimensión, asociándose a un mayor uso de licencia médica ${ }^{5-7}$.

\section{Conclusión}

Basándose en el modelo de sistemas de Betty Neuman, es posible establecer que para que el grupo docente pueda alcanzar la estabilidad, es decir, tenga las condiciones adecuadas para afrontar de manera eficaz todos los elementos estresantes propios de la profesión, se requiere un esfuerzo conjunto tanto por parte de la autoridad educativa (MINEDUC), la que debe orientarse en incrementar sustantivamente los recursos asignados a la educación pública, diseñar políticas de apoyo a la salud docente, establecer estatutos que regulen la sobrecarga laboral, permitir a los educadores participar de las decisiones institucionales y mejorar las condiciones sociales del trabajo docente; también se requiere de la participación de las municipalidades, las que deben gestionar de manera eficaz los recursos asignados por el ministerio al mismo tiempo que realizar y fiscalizar el cumplimiento de las medidas de protección hacia la salud de los educadores, reconocer la labor pedagógica en forma de sueldos apropiados y planes de carrera atractivos, donde la capacitación sea continua y los sistemas de promociones justos y fieles al mérito; contar con el apoyo del gremio docente es importante, ya que ellos son responsables de crear convergencia mediante una organización transparente y democrática, enfocada en levantar conocimiento desde la práctica a fin de contribuir en la evolución y mejora de la educación pública.

La implementación adecuada de estas medidas ayudaría a fortalecer la línea flexible de defensa y la línea de resistencia, así como proporcionar a los educadores herramientas para que ellos mismos sean capaces de desarrollar habilidades que les permitan protegerse de los factores estresantes, a la vez que dichas medidas actuarían directamente sobre los estresores, para resguardar y prevenir que los elementos estresantes crucen la línea normal de defensa y alteren el bienestar docente y por ende, la calidad de la educación impartida.

En este sentido, la calidad de vida en el trabajo tendría el potencial de contribuir en la medición del grado en que los estresores van traspasando cada una de las líneas sistema-sujeto, pues, ya sea que exista una baja satisfacción con alguna dimensión de la CVT o bien con la CVT global, ésta reflejará aspectos concretos sobre los que se debe intervenir a fin de prevenir el desequilibrio o enfermedad, es decir, prevenir que las líneas sean penetradas por estresores, y a su vez, la calidad de la educación se vea en detrimento.

Si bien los hallazgos presentados logran explicarse en base a que los educadores se desempeñan bajo ambientes con gran cantidad de estresores, bajas remuneraciones, formas de contratación inestables, sueldos precarios, condiciones materiales de trabajo insuficientes, no concuerda del todo con la CVT percibida, sólo las dimensiones bienestar logrado a través del trabajo y administración del tiempo libre fueron evaluadas negativamente, mientras que la mayoría de las dimensiones mostraron niveles de satisfacción medio, resultados discrepantes y a la vez congruentes con investigaciones previas.

Es indudable que el desempeño profesional de los educadores se encuentra ligado a las condiciones de trabajo, las que a su vez se reflejan en la CVT, de modo que el deterioro de la calidad de los procesos educativos, se encuentra directamente vinculado a la condición laboral docente, cuantificable mediante la CVT y sus dimensiones. 
Finalmente, Enfermería tiene un rol esencial en el mantenimiento de ambientes laborales saludables, esto específicamente orientado en la promoción y prevención de la salud, lo que a su vez se relaciona directamente con el bienestar de los trabajadores/as, aportes que en el caso de los pedagogos, no sólo contribuirían a reducir la incidencia de patologías de origen crónico, mental y musculo esquelético, sino que también tendrían directa repercusión en el incremento de la calidad de la educación impartida en las instituciones públicas del país.

\section{Responsabilidades Éticas}

Protección de personas y animales. Los autores declaran que en esta investigación no se han realizado experimentos en seres humanos ni en animales.

Confidencialidad. Se resguardó de forma cuidadosa la identidad, confidencialidad y privacidad de cada uno de los participantes

Derecho a la privacidad y consentimiento informado: Los autores han obtenido el consentimiento informado de los participantes.

Financiamiento. Ninguno

Conflicto de intereses. Ninguno

\section{Referencias}

1. Camacaro P. Abordaje conceptual de calidad de vida en el trabajo. Contrib. cienc. soc. Marzo 2010. [Consultado 15 marzo 2016]. Disponible en: https://bit.ly/2AOlTw2

2. Gil-Monte PR. Riesgos psicosociales en el trabajo y salud ocupacional. Rev. Peru. Med. Exp. Salud Pública. 2012; 29 (2): 237-41. [Consultado 15 marzo 2016]. Disponible en: https://bit.ly/2qscPa0

3. Mesén-Fonseca R. Calidad de Vida laboral. Revista de Trabajo Social. 1999; 24 (55): 1146-52. [Consultado 23 octubre 2016]. Disponible en: https://bit.ly/1PI208K

4. Giadrosic-Reyes G. Impacto de la calidad de vida en la disposición del profesor para realizar su trabajo profesional. Docencia. 2002; (18): 74-83. [Consultado 01 diciembre 2016]. Disponible en: https://bit.ly/2qxNJXq

5. Cuenca R, Fabara-Garzón E, Kohen J. Condiciones de trabajo y salud docente: Estudios de Casos en Argentina, Chile, Ecuador, México, Perú y Uruguay. Robalino-Campos M, Körner A (Coord) Santiago de Chile: OREALC/UNESCO; 2005. [Consultado 10 diciembre 2016] Disponible en: https://bit.ly/2DoN9DB

6. Departamento de Bienestar. Informe Estudio de Salud Laboral de los Profesores en Chile. Santiago, Chile: Colegio de Profesores de Chile 2011. [Consultado 10 diciembre 2016]. Disponible en: https://bit.ly/2uuBXhf

7. Cornejo-Chávez R. Condiciones de trabajo y bienestar/malestar docente en profesores de enseñanza media de Santiago de Chile. Educ. Soc. 2009; 30 (107): 409-26. [Consultado 13 noviembre 2017]. Disponible en: https://bit.ly/2PHg1N3

8. EduGlobal \& Profedatos. 1er Censo Docente Chile 2012. EduGlobal Santiago, Chile; 2013. [Consultado 13 noviembre 2016]. Disponible en: https://bit.ly/1K3kdvz

9. Castillo D, Alzamora M. Síndrome de Burnout en docentes que se desempeñan en escuelas públicas vulnerables de la ciudad de Santiago. Akadèmeia. 2015; 13(1): 27-.41. [Consultado 22 noviembre 2016]. Disponible en: https://bit.ly/2PaiYGy

10. Arón AM, Milicic N. Desgaste profesional de los profesores y clima social escolar. Rev. Latinoam. Psicol. 2000; 32 (3): 447-66. [Consultado 26 octubre 2016]. Disponible en: https://bit.ly/2TkdnMb

11. Vidal-Flores C. Profesores municipales con precaria salud mental. Chile: Centro de Investigación y Desarrollo de la Educación, Facultad de Educación-Universidad Alberto Hurtado. 2011. [Consultado 22 noviembre 2016]. Disponible en: https://bit.ly/2QqvPBb 
12. Cornejo-Chávez R, Redondo-Rojo JM. Variables y factores asociados al aprendizaje escolar: Una discusión desde la investigación actual. Estud. pedagóg. 2007.33 (2), 155-75. http://dx.doi.org/10.4067/S0718-07052007000200009

13. Freese BT, Lawson TG. Betty Neuman: Modelo de sistemas. En: Raile-Alligood M, Marriner-Tomey A. Modelos y teorías en enfermería. $7^{\text {a }}$ ed. Barcelona, España: Elsevier; 2011. p.309-34.

14. Consejo Internacional de Enfermeras. Las enfermeras: Una fuerza para el cambio, un recurso vital para la salud. Ginebra, Suiza: International Council of Nurses; 2014.

15. Hernández-Sampieri R, Fernández-Collado C, Baptista-Lucio P. Fundamentos de metodología de la investigación. Madrid, España: McGraw-Hill; 2007.

16. Dirección de Administración de Educación Municipal de Concepción (DAEM). Plan Anual de Desarrollo Educativo (PADEM) 2015. Concepción, Chile: DAEM; 2015.

17. Ilustre Municipalidad de Concepción. Dotación personal DEM y sus remuneraciones año 2015. Santiago, Chile: Portal Transparencia Chile. [Consultado 10 diciembre 2016]. Disponible en: https://bit.ly/2JMe24M

18. González-Baltazar R, Hidalgo-Santacruz G, Salazar JG, et al. Elaboración y Validación del Instrumento para Medir Calidad de Vida en el Trabajo “CVT-GOHISALO”. Cienc. Trab. 2010 ; 12 (36): 332-40. [Consultado 10 diciembre 2016]. Disponible en: https://bit.ly/2yUuuvG

19. González-Baltazar R, Hidalgo-Santacruz G, Salazar-Estrada JG, et al. Instrumento para medir la calidad de vida en el trabajo: CVT-GOHISALO: Manual para su aplicación e interpretación. Guadalajara, México: Instituto de Investigación en Salud Ocupacional; 2009. p. 60.

20. Delgado-García D, Inzulza-González M, Delgado-García F. Calidad de vida en el trabajo: Profesionales de la salud de Clínica Río Blanco y Centro de Especialidades Médicas. Med. segur. trab. 2012; 58 (228): 216-23. http://dx.doi.org/10.4321/S0465-546X2012000300006

21. Emanuel E. ¿Qué hace que la investigación clínica sea ética? Siete requisitos éticos. En: Pellegrini-Filho A, Macklin R (Eds). Investigación en sujetos humanos: Experiencia Internacional. Santiago de Chile: Programa Regional de Bioética, División de Salud y Desarrollo Humano, OPS/ OMS; 1999: 33-46.

22. Silva L, Quintana RM, Reyna D, et al. Nivel de estrés de docentes de enseñanza básica Chillan - Chile. Enfermería en Costa Rica 2006; 27(2): 19-23. [Consultado 27 octubre 2016]. Disponible en: https://bit.ly/2OvrvyL

23. Miño-Sepúlveda A. Clima organizacional y estrés laboral asistencial (Burnout) en profesores de enseñanza media: Un estudio correlacional. Revista PsicologiaCientifica.com [Internet]; 2012; 14(9). Disponible en: https://bit.ly/2PHPrDK

24. Semidober I. Identidad femenina y rol ocupacional. Orientac soc. 2002. 3: 195-203. [Consultado 22 febrero 2017]. Disponible en: https://bit.ly/2D8Vhao

25. Díaz-Andrade E. La desigualdad salarial entre hombres y mujeres: Alcances y limitaciones de la Ley $N^{\circ} 20.348$ para avanzar en justicia de género. Santiago, Chile: Departamento de Estudios de la Dirección del Trabajo, Ministerio del Trabajo y Previsión social. 2015 [consulta 22 febrero 2017]. Disponible en: https://bit.ly/2RGYdz0

26. Ministerio de Educación. Radiografía de los profesores en Chile. Eligeeducar.cl. 2015 [Consultado 26 Febrero 2017]. Disponible en: https://bit.ly/2qD9XXZ

27. Vidal-Araya L. Relaciones de poder en la instalación de la reforma educativa en Chile. Estud. pedagóg. 2012; 38 (1): 333-44. [consulta 22 febrero 2017]. Disponible en: https://bit.ly/2AT7uid

28. Quiceno J, Vinaccia S. La salud en el marco de la psicología de la religión y la espiritualidad. Diversitas 2009; 5(2): 321-36. [Consultado 22 febrero 2017]. Disponible en: https://bit.ly/2JLiG39

29. Ministerio de Salud/Pontificia Universidad Católica de Chile/Universidad Alberto Hurtado. Encuesta Nacional de Salud ENS Chile 2009 - 2010. Santiago, Chile: Ministerio de Salud. 2010. p.4-15. 
30. Rodríguez L, Oramas A, Rodríguez, E. Estrés en docentes de educación básica: estudio de caso en Guanajuato, México. Salud de los Trabajadores 2007; 15(1): 5-16. [Consultado 24 marzo 2016]. Disponible en: https://bit.ly/2A8wrVh

31. Mingote-Adán JC, Del Pino-Cuadrado P, Sánchez-Alaejos R, et al. El trabajador con problemas de salud mental: Pautas generales de detección, intervención y prevención. Med. segur. trab. 2011; 57 (1): 188205. [Consultado 16 febrero 2017]. http://dx.doi.org/10.4321/S0465-546X2011000500012

32. Santana VS. Empleo, condiciones de trabajo y salud. Salud colect 2012; 8 (2): 101-6. [Consultado 25 febrero 2017]. Disponible en: https://bit.ly/2SZdpcu

33. Mingote-Adán JC, Gálvez-Herrer M, Del Pino-Cuadrado P, et al. El paciente que padece un trastorno depresivo en el trabajo. Med. segur. trab. 2009; 55 (214): 41-63. [Consultado 16 febrero 2017]. Disponible en: https://bit.ly/2pBPvpB

34. Sieglin V, Ramos-Tovar ME. Estrés laboral y depresión entre maestros del área metropolitana de Monterrey. Rev. Mex. Sociol. 2007; 69 (3): 517-51. [Consultado 26 febrero 2017]. Disponible en: https://bit.ly/2FaeNGl 